\title{
Traduire
}

Ine autre perspective sur la tataduction

Revue française de la traduction

$233 \mid 2015$

Voyage en équipage

\section{Armand Héroguel, Traduire le néerlandais dans les affaires pénales (Pays-Bas, Belgique)}

Dictionnaire néerlandais-français et français-néerlandais

\section{Benjamin Heyden}

\section{OpenEdition}

\section{Journals}

Édition électronique

URL : http://journals.openedition.org/traduire/771

DOI : 10.4000/traduire.771

ISSN : 2272-9992

Éditeur

Société française des traducteurs

Édition imprimée

Date de publication : 15 décembre 2015

Pagination : 121-128

ISSN : 0395-773X

\section{Référence électronique}

Benjamin Heyden, «Armand Héroguel, Traduire le néerlandais dans les affaires pénales (Pays-Bas, Belgique) », Traduire [En ligne], 233 | 2015, mis en ligne le 15 décembre 2015, consulté le 25 septembre 2020. URL : http://journals.openedition.org/traduire/771 ; DOI : https://doi.org/10.4000/traduire.771 


\section{Compte rendu d'ouvrage \\ Armand Héroguel, Traduire le néerlandais \\ dans les affaires pénales (Pays-Bas, Belgique) \\ Dictionnaire néerlandais-français et français-néerlandais}

\section{Benjamin Heyden}

Armand Héroguel est maître de conférences à l'université de Lille 3 et traducteur judiciaire (expert près la Cour d'appel de Douai depuis 1989). Ainsi qu'il l'explique dans l'introduction, son dictionnaire est " le résultat de 18 ans de traduction pour les tribunaux et de 4 ans de lecture d'ouvrages de doctrine, ainsi que le sous-produit d'une traduction du Code pénal des Pays-Bas " (ce Code fera l'objet d'un prochain compte rendu). S'il le présente en français comme " un dictionnaire terminologique ", " un dictionnaire de définitions et de traductions ", la version néerlandaise de l'introduction dit plus justement : " een vertaal- en verklarend woordenboek " (un dictionnaire traductif et explicatif). On n'y trouve en effet quasiment pas de définitions au sens où on l'entend en terminologie, c'est-à-dire " un microsystème composé de caractères du concept décrit. [...] La définition permet de décrire et de circonscrire le concept traité. Elle $\mathrm{a}$, en terminologie, vocation à être interlinguistique "(1).

Pas ou peu de travail définitoire de cet ordre ici, mais en revanche, de très nombreuses explications ou illustrations des termes en contexte, grâce à des citations (en français et/ou en néerlandais) tirées de la législation ou de la doctrine. Les concepts y sont parfois définis juridiquement mais, plus souvent, les citations proposées permettent simplement d'en inférer le sens. Exemple :

strafklacht $[\mathrm{NL}]$ plainte

Art. 161 Sv. leder die kennis draagt van een begaan strafbaar feit is bevoegd daarvan aangifte of klachte te doen.

Doucet : "La plainte se concrétise en un écrit par lequel une personne, qui se tient pour victime d'une infraction pénale, entend saisir l'autorité judiciaire de ce fait. "

(1) Loïc Depecker, Entre signe et concept - Éléments de terminologie générale, Presses Sorbonne Nouvelle, Paris, 2002, p. 140. En faveur d'Héroguel, reconnaissons que cette vocation interlinguistique a ses limites en terminologie juridique, où les notions (et les définitions) s'enracinent justement dans un système linguistique et juridique particulier. 
Cette nuance posée, poursuivons notre découverte de l'ouvrage : il comporte 4880 entrées (dont 529 sont des renvois vers d'autres entrées) et abonde en informations précieuses sur des termes juridiques propres aux Pays-Bas [NL], à la Belgique [BE] et à l'espace Schengen $[\mathrm{SCH}]$. Le découpage notionnel, par contre, reflète bien l'approche terminologique (vs. lexicographique) : malgré leur identité graphique et leur traduction identique, belaging [BE] harcèlement et belaging [NL] harcèlement sont des termes-notions distincts dans leur droit respectif. Ils font ainsi à juste titre l'objet d'entrées distinctes, alors qu'un dictionnaire classique les aurait réunis sous le même lemme.

Héroguel explique belaging [BE] en citant l'article 442bis du Code pénal belge dans sa version néerlandaise, l'illustre de deux citations de doctrine belge en néerlandais (De Nauw), puis fournit une citation " équivalente " française (Doucet)(2). II explique ensuite belaging [NL] en citant l'article 258b du Code pénal des Pays-Bas et l'article 222-32-2 du Code pénal français, puis l'illustre de deux citations de doctrine française (Gattegno et Doucet).

Les termes synonymes de belaging stalking $[\mathrm{BE}]$ et stalking $[\mathrm{NL}]$ sont aussi traités dans l'ouvrage : les entrées belaging $[\mathrm{BE}]$ et stalking $[\mathrm{BE}]$ sont d'ailleurs absolument identiques (mêmes définitions et mêmes citations), ce qui pose la question de l'utilité de la deuxième, là où un simple renvoi aurait suffi. Les entrées belaging [NL] et stalking [NL] sont quant à elles presque identiques, mais là où la première renvoie à l'article $258 \mathrm{~b}$ du Code pénal néerlandais (qui ne contient pas le terme belaging même, mais le verbe belagen), la deuxième renvoie à l'article 426bis du même Code (qui ne contient pourtant ni stalking, ni stalken).

Signalons au passage quelques autres petites incohérences : l'entrée stalking [BE] signale belaging comme synonyme, mais stalking [NL] ne le signale pas, alors que belaging [NL] existe bien et, comme nous l'avons vu, est traité dans le dictionnaire. Quant aux entrées belaging $[\mathrm{BE}]$ et belaging $[\mathrm{NL}]$, elles ne renvoient pas à stalking. Enfin, ce n'est que grâce à l'index français-néerlandais en fin d'ouvrage que nous avons trouvé l'entrée hinderlijk volgen [NL], qui reçoit exactement le même traitement (mêmes définitions et mêmes citations) que stalking [NL] mais sans renvois mutuels (alors que cela aurait permis de faire l'économie d'une demi-colonne de texte). C'est curieux et un peu regrettable, d'autant que la citation néerlandaise expliquant stalking [NL] ne contient pas le terme stalking mais justement... hinderlijk volgen! Enfin, hinderlijk volgen est traduit par " harcèlement " dans l'index françaisnéerlandais et par " harcèlement moral " dans le corps du dictionnaire (suivi d'une virgule finale : simple erreur typographique ou signe d'une note prévue puis oubliée ?). Ces petites scories seront sans doute corrigées dans une prochaine édition.

(2) On pourrait s'étonner qu'il ne cite pas la version française du Code pénal belge, mais Héroguel, même s'il cite quelques fois certains textes de lois belges, privilégie la traduction en "français de France ". 
Pour mettre à l'épreuve le dictionnaire au-delà de cette première lecture critique, nous l'avons utilisé pour réaliser une traduction qui s'y prêtait à merveille : un mémoire juridique portant sur plusieurs affaires entremêlées, intentées parallèlement en Belgique et aux Pays-Bas, avec, d'une part, plainte pour infractions pénales et constitution de partie civile devant un tribunal de première instance en Belgique et, d'autre part, demande de saisie conservatoire adressée à un juge des référés aux Pays-Bas.

Nous avons pu compter sur le dictionnaire pour traduire correctement et bien cerner les termes suivants (par ordre d'apparition dans notre texte à traduire) :

klacht met burgerlijke partijstelling onderzoeksrechter

gerechtelijk onderzoek

voorzieningenrechter

dagvaarding

vonnis in kort geding

opheffing

beslag plainte avec constitution de partie civile

juge d'instruction

instruction judiciaire

juge des référés

citation

jugement en référé

mainlevée

saisie

En revanche, nous n'y avons pas trouvé rechtbank van eerste aanleg, verwijzingsvonnis, provisionele vordering...

Bien entendu, nul dictionnaire ne peut prétendre à l'exhaustivité, d'autant que celui-ci est spécialisé dans un sous-domaine du droit, mais signalons à titre de comparaison que ces termes sont mentionnés dans le dictionnaire Moors(3).

Quant à la notion de strafbaar feit, si nous avons trouvé pour elle trois solutions dans le dictionnaire :

\section{strafbaar feit $[\mathrm{BE}]$ fait répréhensible}

strafbaar feit [NL] infraction

\section{strafbaar feit $[\mathrm{SCH}]$ fait punissable}

nous avons finalement choisi de la traduire par "fait qualifié par la loi pénale ". II est vrai que cette dernière traduction était appelée par le contexte particulier de notre document et la volonté de désigner par ce terme unique une réalité pénale belge et une réalité pénale néerlandaise.

(3) Joseph Moors \& Siegfried Theissen, Juridisch woordenboek Nederlands-Frans/Dictionnaire juridique françaisnéerlandais, 6e édition, Die Keure/La Charte, Bruxelles, 2006. 
II n'en faut pas moins souligner la richesse de ce dictionnaire. Pour l'élaborer, l'auteur a épluché une série impressionnante de sources de référence, dont il a extrait des informations juridiques solides sur les droits pénaux néerlandais, belge, français et européen. II a en outre signalé de nombreuses relations d'antonymie, de synonymie, d'hyperonymie ou d'hyponymie qui aident à cerner les concepts et donnent aussi envie aux lecteurs d'approfondir la lecture. Enfin, le dictionnaire est parsemé de "remarques " apportant des précisions culturelles et juridiques qui raviront les comparatistes. En voici quelques exemples :

- Pour Meer- en Vaart-verweer moyen visant l'absence de motivation au regard des moyens de preuve

Rem. Du nom d'un arrêt de la Cour de cassation néerlandaise.

- Pour politiesepot abandon des poursuites par les forces de police

Rem. En France, le classement sans suite est un acte du parquet.

- Pour rolzitting audience de mise en état

Rem. En France, ce procédé n'est mentionné que pour le civil.

- Pour rijkswacht gendarmerie

Rem. Supprimée en Belgique le 1er avril 2001.

- Pour straatschenderij vandalisme

Rem. Correspondance partielle.

Ce dictionnaire est technique et foisonnant, sa mise en page est soignée et son index françaisnéerlandais est une aide précieuse. Les traducteurs néerlandais-français spécialisés dans la langue juridique, en particulier s'ils traitent des textes à cheval sur plusieurs systèmes juridiques, lui feront une place dans leur bibliothèque.

benjamin.heyden@ec.europa.eu

HÉROGUEL Armand, Traduire le néerlandais dans les affaires pénales (Pays-Bas, Belgique) Dictionnaire néerlandais-français et français-néerlandais, Presses universitaires du Septentrion, Villeneuve-d'Ascq, 2014.

Benjamin Heyden est traducteur-terminologue à la Commission européenne et Maître de conférences à l'Université de Liège (Belgique). 